\title{
Canadian results from the European Men-who- have-sex-with-men Internet survey (EMIS-2017)
}

\author{
N Brogan ${ }^{1}$, DM Paquette ${ }^{1 *}$, NJ Lachowsky², M Blais ${ }^{3}$, DJ Brennan ${ }^{4}$, TA Hart ${ }^{5}$, B Adam ${ }^{6,7}$
}

\begin{abstract}
Background: In 2017, the international European Men-who-have-sex-with-men Internet Survey (EMIS-2017) collected data from 50 countries, including Canada for the first time.
\end{abstract}

Objective: To provide an overview of the Canadian EMIS-2017 data to describe the sexually transmitted and other bloodborne infection (STBBI) related needs of gay, bisexual and other men who have sex with men (gbMSM).

Methods: The EMIS-2017 questionnaire was an updated version of EMIS-2010. It included self-reported sociodemographic data, experience of discrimination, mental health and substance use, knowledge of preexposure prophylaxis (PrEP) for HIV, sexual practices and history of STBBI testing and diagnosis. Analysis was largely descriptive.

Results: Of the 6,059 respondents from Canada, 5,165 participants met the inclusion criteria for this analysis. The majority of participants were born in Canada (79.3\%); and over half of the respondents (56.7\%) were under the age of 39. In terms of discrimination related to their attraction to other men, participants reported high levels of intimidation (31.9\%), verbal abuse (22.1\%) and physical violence (1.5\%) in the previous year. Regarding mental health, $23.9 \%$ had a moderate to severe depression/anxiety score. Almost two-thirds (64.1\%) indicated substance use and one-fifth (21.5\%) reported chemsex (or the use of stimulant drugs to make sex more intense or last longer). Only $8.4 \%$ of participants reported use of PrEP for HIV; however, $51.7 \%$ reported being likely to use PrEP if it was available and affordable. Sexual practices, such as condom use, varied by PrEP use with $91.3 \%$ of men using PrEP reporting condomless anal intercourse (CAI) compared with $71.5 \%$ of men not on PrEP. In terms of STBBI testing, $1.5 \%$ reported being diagnosed with hepatitis $C$ and $9.0 \%$ reported an HIV diagnosis. Of those with an HIV diagnosis, most were on treatment $(99.1 \%)$ and had an undetectable viral load $(96.7 \%)$.

Conclusion: gbMSM in Canada experienced stigma, discrimination and mental health problems; substance use was high as were high-risk sexual practices, such as CAI, among some groups of men. There was a gap between the proportion of men who were interested in PrEP and those who actually used it; and comprehensive STBBI testing was low.

These findings can inform public health action and provide a baseline to examine the impact of current and new interventions.
This work is licensed under a Creative Commons Attribution 4.0 Internationa License.

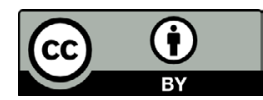

Affiliations

${ }^{1}$ Public Health Agency of Canada, Ottawa, ON

2 University of Victoria, Victoria, BC

${ }^{3}$ Université du Québec à Montréal, Montréal, QC

${ }^{4}$ University of Toronto, Toronto, ON

${ }^{5}$ Ryerson University and University of Toronto, Toronto, ON

${ }^{6}$ University of Windsor, Windsor, ON

${ }^{7}$ Ontario HIV Treatment Network, Toronto, ON

${ }^{\star}$ Correspondence:

dana.paquette@canada.ca

Suggested citation: Brogan N, Paquette DM, Lachowsky NJ, Blais M, Brennan DJ, Hart TA, Adam B. Canadian results from the European Men-who-have-sex-with-men Internet survey (EMIS-2017). Can Commun Dis Rep 2019;45(11):271-82. https://doi.org/10.14745/ccdr.v45i11a01

Keywords: HIV, sexually transmitted infections, sexual risk practices, gay bisexual and other men who have sex with men, gbMSM, preexposure prophylaxis, postexposure prophylaxis

\section{Introduction}

In 2017, the second iteration of the European Men-who-havesex-with-men Internet Survey (EMIS-2017) was launched (1). This survey collected data from gay, bisexual and other men who have sex with men (gbMSM) living in 50 countries, with the aim of generating data useful for planning HIV and sexually transmitted infection (STI) prevention and care programs, and monitoring of 
progress in these areas (1). Canada participated in this survey for the first time.

In Canada, gbMSM continue to be particularly affected by sexually transmitted and bloodborne infections (STBBIs). In 2016, it was estimated that gbMSM make up more than half (52.5\%) of the population living with HIV infection in Canada (2). Rates of syphilis and gonorrhea have increased over the past several years, with the increase among men largely in the gbMSM population in Canada (3-5) and internationally $(6,7)$. Hepatitis C is also on the rise among gbMSM who are HIV-positive (8).

The reasons for this are multifactorial. Among HIV-negative men, new preventive interventions like preexposure prophylaxis (PrEP) in combination with reduced use of condoms may, in part, contribute to the rise in STIs other than HIV $(3,4)$. Among HIV-positive men, serosorting and knowledge of an undetectable viral load may lead to minimizing the risk of HIV transmission, which can lead to a concurrent decreased use of condoms, thus increasing the risk of other STBBIs $(3,4)$. Other factors that could be influencing rates of STBBls include changing community norms and the use of illicit drugs to enhance sexual experiences (i.e. party and play/chemsex) (9). Structural factors, such as stigma related to sexual orientation and to HIV infection, a lack of provider knowledge and training in gbMSM sexual health risks and needs, and issues related to cost and access to PrEP and HIV treatment may also be contributing to the STBBI burden among gbMSM (10).

Gathering national-level information on risk and prevention behaviours, health service needs and health outcomes is crucial for understanding the current trends and for guiding the planning and evaluation of public health interventions to prevent STBBls among gbMSM. The objective of this report is to provide an overview of the EMIS-2017 data from Canada.

\section{Methods}

EMIS-2017 was undertaken by Sigma Research at the London School of Hygiene and Tropical Medicine in association with the Robert Koch Institute in Berlin. The survey was funded by the European Union Health Programme 2014-2020 for Europe. The survey ran from October 2017 to January 2018, inclusively, across 50 countries. In Canada, the Public Health Agency of Canada (PHAC) funded the promotion of the survey to Canadian gbMSM. Community-involved researchers (NJL, MB, DJB, TAH and $\mathrm{BA}$ ), along with two PHAC representatives, provided feedback on the questionnaire and recruitment methods and a review and interpretation of the results.

While EMIS uses the term "men who have sex with men (MSM)", the authors use the term "gbMSM" to describe the same population as the latter is commonly used in Canada. A more detailed description of the methods can be found elsewhere (11).

\section{Questionnaire}

The EMIS-2017 survey was based on the questionnaire successfully used in EMIS-2010. Updates were based on a review of evidence of the epidemiology of HIV infection and STIs; the STBBI-related risk and precautionary behaviours of gbMSM; a policy and practice mapping exercise; a scoping exercise of available gbMSM questionnaires published since EMIS-2010; and three rounds of consultations with partner countries, including Canada.

The final version of the questionnaire included questions on:

- Sociodemographic characteristics of EMIS participants

- Experiences of discrimination, mental health and substance use

- Knowledge and use of postexposure prophylaxis (PEP) and PrEP

- Sexual practices - information on chemsex was captured by asking about the use of stimulant drugs (including ecstasy/ MDMA, cocaine, amphetamine, crystal methamphetamine, mephedrone and ketamine to make sex feel more intense or last longer)

- $\quad$ STBBI testing and diagnosis

The survey was available in 33 languages simultaneously, including 22 of the 23 official languages of the European Union; Canadian researchers provided edits to both the French and English version and the questionnaire was piloted among a small group of Canadian gbMSM. The EMIS-2017 questionnaire can be found online (12).

\section{Recruitment}

Sigma staff commissioned advertising from 10 multi-country "dating" platforms including PlanetRomeo, Grindr, Hornet, Qruiser, RECON, Scruff, Gaydar, Manhunt/Jack'd, GROWLr and Bluesystem. Within Canada, advertisements and banners on social media, gay news websites and sexual networking apps were posted. Electronic and offline promotional materials were provided to community-based organizations across Canada, such as the Health Initiative for Men, for distribution to their networks.

All online promotions of EMIS, paid or unpaid, were allocated a specific URL to direct potential participants to the EMIS landing page. Here they could make their language selection and proceed to the survey; this page also captured data on the recruitment source. Unfortunately, the Grindr Source codes were erroneously labelled in Canada; during data cleaning, these were rectified as much as possible using the timeline for participation invitations sent out by Grindr.

\section{Consent and inclusion criteria}

Before proceeding to the survey, participants in Canada had to confirm that they had read and understood the nature and purpose of the study, wanted to participate and were aged 16 years or older. Inclusion criteria included: 
- Living in one of 50 countries participating in the study

- Identifying as a man, including cisgender men (i.e. individuals assigned male at birth who identify as men) and transgender men (i.e. individuals assigned female at birth who identify as men)

- Being sexually attracted to men and/or ever having had sex with men

For the purposes of this analysis, participants needed to provide the first part of their postal code so they could be assigned to a province or territory. Three discrepancy flags were created with regard to age, steady male partners and non-steady partners. Participants with inconsistent data were excluded from this analysis.

\section{Analysis}

Descriptive analyses (frequencies and proportions) were conducted. Participants' characteristics were described by province and territory. Due to sample sizes less than 10, men who resided in Yukon were combined with those living in British Columbia, those from Northwest Territories with Alberta and those from Nunavut with Ontario. Similarly, participants from Manitoba and Saskatchewan were combined as were those from the four Atlantic Provinces.

A combined measure of anxiety and depression was calculated using a validated brief screening scale for anxiety and depression, the Patient Health Questionnaire-4 (13). The CAGE 4-item questionnaire was used as an indicator of alcohol dependency (14).

To monitor the uptake and effect of new HIV prevention strategies, the following indicators were derived:

- Anal intercourse with casual partners

- Condom use with casual partners

- Any condomless anal intercourse (CAI) with casual partners in the last 12 months by HIV-positive men on HIV treatment and with an undetectable viral load

- Any CAI with casual partners in the last 12 months by HIV-negative men on PrEP

- $\quad$ Any CAl with casual partners in the last 12 months by HIV-positive men not on HIV treatment or with a detectable viral load

- $\quad$ Any CAl with casual partners in the last 12 months by HIV-negative or untested men not on PrEP (15). Casual partners were defined in the questionnaire as non-steady partners: "men you have had sex with once only, and men you have sex with more than once but who you don't think of as a steady partner"

"Full STI screening" was a composite variable indicating testing for HIV infection, a blood test, an anal swab and a urethral swab (or a vaginal swab or a urine test) in non-HIV-diagnosed respondents in the previous 12 months. Only non-HIV-diagnosed respondents were included in this indicator to ensure that it was not influenced by counts from HIV-diagnosed men who typically have regular routine STI checks as part of their clinical follow-up. This variable was developed for cross-country comparison of STI testing and treatment services coverage in the Dublin Declaration Monitoring (16). Although pharyngeal swabs are recommended in Canada (17), EMIS-2017 did not collect this information.

Numbers and proportions were suppressed when the numerator was less than five and the denominator was less than 100.

\section{Results}

A total of 6,059 Canadian gbMSM participated in EMIS-2017. Of these, 894 (14.8\%) were removed because they had discrepant data or did not provide a forward sortation area (i.e. first three characters of their postal code) and so could not be categorized by province/territory. The remaining 5,165 participants from Canada were included in this analysis.

\section{Characteristics of EMIS participants}

All provinces and territories were represented, with $23.1 \%$ from British Columbia and Yukon, 13.7\% from Alberta and the Northwest Territories, $6.6 \%$ from Saskatchewan and Manitoba, $33.0 \%$ from Ontario and Nunavut, 15.3\% from Quebec and $8.3 \%$ from the Atlantic Provinces. Nationally, the majority of participants completed the survey in English (91.0\%), with $7.4 \%$ completing it in French and the $1.6 \%$ completing it in one of 19 other languages.

Over $50 \%$ of participants were under the age of $39(56.7 \%)$ (Table 1). The median age of the population was 36 years of age. The majority of the participants identified as gay $(76.0 \%)$, and the remaining participants identified as bisexual (17.5\%), straight $(0.9 \%)$ or with another term or no term $(5.7 \%)$. Of the participants, $2.4 \%$ identified as transgender men. In terms of ethnicity, 3.8\% identified as Indigenous, 3.8\% as Latin American, $3.1 \%$ identified as Asian, 2.8\% as East or Southeast Asian, 1.9\% as South Asian, $1.7 \%$ as Black and $1.4 \%$ as Arab/West Asian. One-fifth (20.7\%) of the participants were born outside of Canada.

Over three-quarters $(78.5 \%)$ of the participants reported having four or more years of education past the age of 16. Almost three-quarters $(71.0 \%)$ reported that they were employed (including full-time, part-time and self-employed) whereas $13.2 \%$ identified as students, and the remaining $15.8 \%$ reported being unemployed, retired (including medically), on long-term sick leave or other. Almost half (47.9\%) of the participants reported being comfortable in their financial situation, $31.9 \%$ reported being neither comfortable nor struggling, and one-fifth (20.2\%) reported being uncomfortable. 
Table 1: Sociodemographic characteristics of Canadian participants in the EMIS-2017 $(N=5,165)$

\begin{tabular}{|c|c|c|c|c|c|c|c|c|c|c|c|c|c|c|}
\hline \multirow[t]{2}{*}{ Characteristics } & \multicolumn{2}{|c|}{$\begin{array}{c}\text { British } \\
\text { Columbia }+ \\
\text { Yukon } \\
(n=1,191)^{\mathrm{a}}\end{array}$} & \multicolumn{2}{|c|}{$\begin{array}{c}\text { Alberta + } \\
\text { Northwest } \\
\text { Territories } \\
(n=710)^{\mathrm{a}}\end{array}$} & \multicolumn{2}{|c|}{$\begin{array}{c}\text { Saskatchewan } \\
+ \\
\text { Manitoba } \\
(n=339)^{a}\end{array}$} & \multicolumn{2}{|c|}{$\begin{array}{c}\text { Ontario } \\
+ \\
\text { Nunavut } \\
(n=1,707)^{\mathrm{a}}\end{array}$} & \multicolumn{2}{|c|}{$\begin{array}{l}\text { Quebec } \\
(n=789)^{a}\end{array}$} & \multicolumn{2}{|c|}{$\begin{array}{l}\text { Atlantic } \\
\text { Provinces } \\
(n=429)^{a}\end{array}$} & \multicolumn{2}{|c|}{$\begin{array}{c}\text { Total } \\
(\mathrm{N}=5,165)^{\mathrm{a}}\end{array}$} \\
\hline & $\mathrm{n}$ & $\%$ & $\mathrm{n}$ & $\%$ & $n$ & $\%$ & $\mathrm{n}$ & $\%$ & $\mathrm{n}$ & $\%$ & $n$ & $\%$ & $n$ & $\%$ \\
\hline \multicolumn{15}{|l|}{ Age category } \\
\hline $16-24$ & 160 & 13.4 & 148 & 20.8 & 63 & 18.6 & 281 & 16.5 & 165 & 20.9 & 89 & 20.7 & 906 & 17.5 \\
\hline $25-39$ & 492 & 41.3 & 295 & 41.5 & 137 & 40.4 & 607 & 35.6 & 334 & 42.3 & 161 & 37.5 & 2,026 & 39.2 \\
\hline $40-54$ & 326 & 27.4 & 166 & 23.4 & 83 & 24.5 & 526 & 30.8 & 179 & 22.7 & 96 & 22.4 & 1,376 & 26.6 \\
\hline $55-69$ & 193 & 16.2 & 90 & 12.7 & 53 & 15.6 & 250 & 14.6 & 102 & 12.9 & 77 & 17.9 & 765 & 14.8 \\
\hline $70+$ & 20 & 1.7 & 11 & 1.5 & 3 & 0.88 & 43 & 2.5 & 9 & 1.1 & 6 & 1.4 & 92 & 1.8 \\
\hline \multicolumn{15}{|l|}{ Sexual identity } \\
\hline Gay or homosexual & 975 & 82.0 & 503 & 70.8 & 223 & 65.8 & 1,294 & 75.8 & 627 & 79.5 & 302 & 70.4 & 3,924 & 76.0 \\
\hline Bisexual & 138 & 11.6 & 156 & 22.0 & 98 & 28.9 & 297 & 17.4 & 116 & 14.7 & 96 & 22.4 & 901 & 17.5 \\
\hline Straight or heterosexual & 4 & 0.3 & 12 & 1.7 & 6 & 1.8 & 17 & 1.0 & 4 & 0.5 & 2 & 0.5 & 45 & 0.9 \\
\hline Other & 72 & 6.1 & 39 & 5.5 & 12 & 3.5 & 99 & 5.8 & 42 & 5.3 & 29 & 6.8 & 293 & 5.7 \\
\hline \multicolumn{15}{|l|}{ Gender identity } \\
\hline Cisgender man & 1,158 & 97.2 & 690 & 97.2 & 331 & 97.6 & 1,675 & 98.1 & 772 & 97.8 & 413 & 96.3 & 5,039 & 97.6 \\
\hline Transgender man & 33 & 2.8 & 20 & 2.8 & 8 & 2.4 & 32 & 1.9 & 17 & 2.2 & 16 & 3.7 & 126 & 2.4 \\
\hline \multicolumn{15}{|c|}{ Proportion of friends, family who know about attraction to men } \\
\hline All, almost all & 652 & 55.1 & 291 & 41.7 & 139 & 41.5 & 819 & 48.6 & 432 & 55.0 & 203 & 47.4 & 2,536 & 49.6 \\
\hline More than half & 227 & 19.2 & 125 & 17.9 & 52 & 15.5 & 318 & 18.9 & 124 & 15.8 & 60 & 14.0 & 906 & 17.7 \\
\hline Less than half & 136 & 11.5 & 80 & 11.5 & 44 & 13.1 & 182 & 10.8 & 74 & 9.4 & 54 & 12.6 & 570 & 11.1 \\
\hline Few & 131 & 11.1 & 135 & 19.3 & 71 & 21.2 & 255 & 15.1 & 100 & 12.7 & 79 & 18.5 & 771 & 15.1 \\
\hline None & 38 & 3.2 & 67 & 9.6 & 29 & 8.7 & 110 & 6.5 & 55 & 7.0 & 32 & 7.5 & 331 & 6.5 \\
\hline \multicolumn{15}{|l|}{ Born in Canada } \\
\hline No & 307 & 25.8 & 99 & 14.0 & 38 & 11.2 & 396 & 23.2 & 190 & 24.1 & 40 & 9.3 & 1,070 & 20.7 \\
\hline Yes & 881 & 74.2 & 610 & 86.0 & 301 & 88.8 & 1,309 & 76.8 & 598 & 75.9 & 388 & 90.7 & 4,087 & 79.3 \\
\hline \multicolumn{15}{|l|}{ Ethnicity } \\
\hline White & 795 & 74.2 & 507 & 80.9 & 237 & 78.0 & 1,151 & 76.5 & 524 & 78.9 & 306 & 87.7 & 3,520 & 77.9 \\
\hline Indigenous & 46 & 4.3 & 34 & 5.4 & 24 & 7.9 & 35 & 2.3 & 20 & 3.0 & 11 & 3.2 & 170 & 3.8 \\
\hline Latin American & 39 & 3.6 & 16 & 2.6 & 10 & 3.3 & 53 & 3.5 & 41 & 6.2 & 1 & 0.3 & 160 & 3.5 \\
\hline Asian & 47 & 4.4 & 17 & 2.7 & 8 & 2.6 & 53 & 3.5 & 12 & 1.8 & 3 & 0.9 & 140 & 3.1 \\
\hline East/Southeast Asian & 57 & 5.3 & 16 & 2.6 & 5 & 1.6 & 36 & 2.4 & 8 & 1.2 & 4 & 1.1 & 126 & 2.8 \\
\hline South Asian & 22 & 2.1 & 10 & 1.6 & 4 & 1.3 & 45 & 3.0 & 5 & 0.8 & 2 & 0.6 & 88 & 1.9 \\
\hline Black $^{b}$ & 10 & 0.9 & 6 & 1.0 & 5 & 1.6 & 43 & 2.9 & 7 & 1.1 & 6 & 1.7 & 77 & 1.7 \\
\hline Arab/West Asian & 13 & 1.2 & 5 & 0.8 & 2 & 0.7 & 22 & 1.5 & 19 & 2.9 & 4 & 1.1 & 65 & 1.4 \\
\hline Other & 42 & 3.9 & 16 & 2.6 & 9 & 3.0 & 67 & 4.5 & 28 & 4.2 & 12 & 3.4 & 174 & 3.8 \\
\hline \multicolumn{15}{|c|}{ Years of education past 16 years of age } \\
\hline None & 113 & 9.5 & 70 & 9.9 & 37 & 10.9 & 174 & 10.2 & 50 & 6.3 & 39 & 9.1 & 483 & 9.4 \\
\hline $1-3$ & 156 & 13.1 & 124 & 17.5 & 49 & 14.5 & 168 & 9.8 & 66 & 8.4 & 65 & 15.2 & 628 & 12.2 \\
\hline $4-6$ & 322 & 27.0 & 210 & 29.6 & 116 & 34.2 & 479 & 28.1 & 193 & 24.5 & 136 & 31.7 & 1,456 & 28.2 \\
\hline $7+$ & 600 & 50.4 & 306 & 43.1 & 137 & 40.4 & 886 & 51.9 & 480 & 60.8 & 189 & 44.1 & 2,598 & 50.3 \\
\hline Occupation & & & & & & & & & & & & & & \\
\hline Employed full-time & 664 & 55.8 & 394 & 55.5 & 206 & 60.8 & 944 & 55.5 & 422 & 53.6 & 228 & 53.1 & 2,858 & 55.4 \\
\hline Employed part-time & 80 & 6.7 & 59 & 8.3 & 17 & 5.0 & 109 & 6.4 & 58 & 7.4 & 41 & 9.6 & 364 & 7.1 \\
\hline Self-employed & 110 & 9.3 & 53 & 7.5 & 26 & 7.7 & 154 & 9.0 & 66 & 8.4 & 29 & 6.8 & 438 & 8.5 \\
\hline Unemployed & 70 & 5.9 & 45 & 6.3 & 22 & 6.5 & 101 & 5.9 & 31 & 3.9 & 21 & 4.9 & 290 & 5.6 \\
\hline Student & 125 & 10.5 & 88 & 12.4 & 40 & 11.8 & 214 & 12.6 & 155 & 19.7 & 60 & 14.0 & 682 & 13.2 \\
\hline Retired & 84 & 7.1 & 41 & 5.8 & 18 & 5.3 & 105 & 6.2 & 41 & 5.2 & 34 & 7.9 & 323 & 6.3 \\
\hline Other & 56 & 4.7 & 30 & 4.2 & 10 & 2.9 & 75 & 4.4 & 14 & 1.8 & 16 & 3.7 & 201 & 3.9 \\
\hline Comfort level with current income & & & & & & & & & & & & & & \\
\hline Living really comfortably & 190 & 16.0 & 108 & 15.2 & 50 & 14.7 & 250 & 14.7 & 144 & 18.3 & 64 & 14.9 & 806 & 15.6 \\
\hline Living comfortably & 374 & 31.4 & 207 & 29.2 & 116 & 34.2 & 572 & 33.6 & 281 & 35.7 & 118 & 27.5 & 1,668 & 32.3 \\
\hline Neither comfortable nor struggling & 387 & 32.5 & 244 & 34.4 & 106 & 31.3 & 530 & 31.1 & 234 & 29.7 & 145 & 33.8 & 1,646 & 31.9 \\
\hline Struggling & 170 & 14.3 & 102 & 14.4 & 42 & 12.4 & 235 & 13.8 & 105 & 13.3 & 76 & 17.7 & 730 & 14.2 \\
\hline Really struggling & 69 & 5.8 & 49 & 6.9 & 25 & 7.4 & 115 & 6.8 & 23 & 2.9 & 26 & 6.1 & 307 & 6.0 \\
\hline
\end{tabular}




\section{Experiences of discrimination, mental health and substance use}

In the year previous to the survey, $31.9 \%$ of participants reported experiencing intimidation, $22.1 \%$ reported experiencing verbal abuse and $1.5 \%$ reported experiencing physical violence related to knowledge or presumption of attraction to men (Table 2).
Almost one-quarter (23.9\%) of participants were classified as moderate to severe on a combined measure of anxiety and depression, and $26.1 \%$ of participants reported some suicidal ideation ranging from on occasion to nearly every day in the previous two weeks.

With regard to substance use, $64.1 \%$ indicated ever using any illicit substance. The most frequently reported drugs used included cannabis (46.6\%), which was not yet legal at the

Table 2: Experiences of discrimination related to knowledge or presumption of attraction to men, mental health and substance use of Canadian participants in the EMIS-2017 $(\mathrm{N}=5,165)$

\begin{tabular}{|c|c|c|c|c|c|c|c|c|c|c|c|c|c|c|}
\hline \multirow[t]{2}{*}{ Characteristics } & \multicolumn{2}{|c|}{$\begin{array}{c}\text { British Columbia } \\
\quad+\text { Yukon } \\
(n=1,191)^{\mathrm{a}}\end{array}$} & \multicolumn{2}{|c|}{$\begin{array}{c}\text { Alberta + } \\
\text { Northwest } \\
\text { Territories } \\
(n=710)^{\mathrm{a}}\end{array}$} & \multicolumn{2}{|c|}{$\begin{array}{c}\text { Saskatchewan + } \\
\text { Manitoba } \\
(n=339)^{\mathrm{a}}\end{array}$} & \multicolumn{2}{|c|}{$\begin{array}{c}\text { Ontario } \\
+ \\
\text { Nunavut } \\
(n=1,707)^{\mathrm{a}}\end{array}$} & \multicolumn{2}{|c|}{$\begin{array}{l}\text { Quebec } \\
(n=789)^{a}\end{array}$} & \multicolumn{2}{|c|}{$\begin{array}{l}\text { Atlantic Provinces } \\
\qquad(n=429)\end{array}$} & \multicolumn{2}{|c|}{$\begin{array}{c}\text { Total } \\
(\mathrm{N}=5,165)^{\mathrm{a}}\end{array}$} \\
\hline & n & $\%$ & $n$ & $\%$ & $n$ & $\%$ & $\mathbf{n}$ & $\%$ & \begin{tabular}{l|l}
$\mathbf{n}$ & \\
\end{tabular} & $\%$ & $\mathbf{n}$ & $\%$ & $\mathbf{n}$ & $\%$ \\
\hline \multicolumn{15}{|c|}{ Homophobic intimidation and assault started at or intimidated } \\
\hline Within last six months & 172 & 14.5 & 94 & 13.3 & 43 & 12.7 & 233 & 13.7 & 67 & 8.5 & 38 & 8.9 & 647 & 12.6 \\
\hline Six months to a year ago & 249 & 20.9 & 145 & 20.5 & 69 & 20.4 & 306 & 18.0 & 135 & 17.2 & 89 & 20.7 & 993 & 19.3 \\
\hline $1-5$ years ago & 190 & 16.0 & 96 & 13.5 & 36 & 10.6 & 240 & 14.1 & 115 & 14.6 & 60 & 14.0 & 737 & 14.3 \\
\hline $5+$ years ago & 257 & 21.6 & 104 & 14.7 & 49 & 14.5 & 351 & 20.6 & 184 & 23.4 & 80 & 18.6 & 1,025 & 19.9 \\
\hline Never & 321 & 27.0 & 270 & 38.1 & 142 & 41.9 & 570 & 33.5 & 286 & 36.3 & 162 & 37.8 & 1,751 & 34.0 \\
\hline \multicolumn{15}{|l|}{ Verbal insults ${ }^{\mathrm{b}}$} \\
\hline Within last six months & 77 & 6.5 & 57 & 8.1 & 19 & 5.6 & 98 & 5.8 & 35 & 4.5 & 18 & 4.2 & 304 & 5.9 \\
\hline Six months to a year ago & 223 & 18.8 & 113 & 16.0 & 62 & 18.3 & 289 & 17.0 & 83 & 10.6 & 64 & 15.0 & 834 & 16.2 \\
\hline $1-5$ years ago & 265 & 22.3 & 117 & 16.5 & 60 & 17.7 & 332 & 19.5 & 149 & 19.0 & 73 & 17.1 & 996 & 19.4 \\
\hline $5+$ years ago & 323 & 27.2 & 137 & 19.4 & 70 & 20.6 & 460 & 27.1 & 219 & 27.9 & 122 & 28.5 & 1,331 & 25.9 \\
\hline Never & 301 & 25.3 & 283 & 40.0 & 128 & 37.8 & 520 & 30.6 & 299 & 38.1 & 151 & 35.3 & 1,682 & 32.7 \\
\hline \multicolumn{15}{|l|}{ Punched, hit, kicked or beaten } \\
\hline Within last six months & 4 & 0.3 & 8 & 1.1 & 1 & 0.3 & 4 & 0.2 & 1 & 0.1 & 4 & 0.9 & 22 & 0.4 \\
\hline Six months to a year ago & 17 & 1.4 & 10 & 1.4 & 5 & 1.5 & 11 & 0.6 & 7 & 0.9 & 6 & 1.4 & 56 & 1.1 \\
\hline $1-5$ years ago & 40 & 3.4 & 40 & 5.6 & 13 & 3.8 & 50 & 2.9 & 23 & 2.9 & 13 & 3.0 & 179 & 3.5 \\
\hline $5+$ years ago & 216 & 18.2 & 83 & 11.7 & 34 & 10.0 & 292 & 17.2 & 125 & 15.9 & 71 & 16.6 & 821 & 15.9 \\
\hline Never & 913 & 76.7 & 568 & 80.1 & 286 & 84.4 & 1,342 & 79.0 & 630 & 80.2 & 334 & 78.0 & 4,073 & 79.1 \\
\hline \multicolumn{15}{|l|}{ Anxiety and Depression Scale ${ }^{c}$} \\
\hline Normal & 472 & 40.0 & 276 & 39.1 & 137 & 40.7 & 731 & 43.4 & 321 & 41.3 & 188 & 44.7 & 2,125 & 41.6 \\
\hline Mild & 393 & 33.3 & 249 & 35.3 & 116 & 34.4 & 546 & 32.4 & 308 & 39.6 & 149 & 35.4 & 1,761 & 34.5 \\
\hline Moderate & 176 & 14.9 & 92 & 13.0 & 41 & 12.2 & 245 & 14.5 & 87 & 11.2 & 44 & 10.5 & 685 & 13.4 \\
\hline Severe & 139 & 11.8 & 88 & 12.5 & 43 & 12.8 & 164 & 9.7 & 61 & 7.9 & 40 & 9.5 & 535 & 10.5 \\
\hline \multicolumn{15}{|c|}{ Suicidal ideation in previous two weeks } \\
\hline Not at all & 885 & 74.4 & 510 & 71.8 & 237 & 70.1 & 1,268 & 74.7 & 592 & 75.2 & 311 & 72.5 & 3,803 & 73.8 \\
\hline Some days & 200 & 16.8 & 129 & 18.2 & 70 & 20.7 & 305 & 18.0 & 148 & 18.8 & 87 & 20.3 & 939 & 18.2 \\
\hline More than half the days & 51 & 4.3 & 33 & 4.6 & 10 & 3.0 & 63 & 3.7 & 20 & 2.5 & 16 & 3.7 & 193 & 3.7 \\
\hline Nearly every day & 54 & 4.5 & 38 & 5.4 & 21 & 6.2 & 62 & 3.7 & 27 & 3.4 & 15 & 3.5 & 217 & 4.2 \\
\hline \multicolumn{15}{|l|}{ Alcohol dependency ${ }^{d}$} \\
\hline Not alcohol dependent & 936 & 79.1 & 553 & 78.4 & 262 & 77.7 & 1,345 & 79.7 & 629 & 80.5 & 363 & 85.6 & 4,088 & 79.9 \\
\hline Alcohol dependent & 248 & 20.9 & 152 & 21.6 & 75 & 22.3 & 343 & 20.3 & 152 & 19.5 & 61 & 14.4 & 1,031 & 20.1 \\
\hline \multicolumn{15}{|c|}{ Ever taken recreational or illicit drugs } \\
\hline No & 367 & 31.1 & 254 & 35.9 & 151 & 44.7 & 597 & 35.3 & 294 & 37.4 & 176 & 41.6 & 1,839 & 35.9 \\
\hline Yes & 812 & 68.9 & 454 & 64.1 & 187 & 55.3 & 1,092 & 64.7 & 492 & 62.6 & 247 & 58.4 & 3,284 & 64.1 \\
\hline \multicolumn{15}{|l|}{ Illicit drugs used in previous year } \\
\hline Cannabis & 609 & 51.7 & 326 & 46.0 & 131 & 38.8 & 797 & 47.2 & 333 & 42.5 & 192 & 45.4 & 2,388 & 46.6 \\
\hline Cocaine & 192 & 16.3 & 98 & 13.9 & 39 & 11.5 & 222 & 13.2 & 123 & 15.6 & 40 & 9.5 & 714 & 14 \\
\hline Ecstasy pills & 152 & 12.9 & 53 & 7.5 & 15 & 4.4 & 147 & 8.7 & 77 & 9.5 & 16 & 3.8 & 458 & 8.9 \\
\hline $\begin{array}{l}\text { Ecstasy in the form of a crystal or } \\
\text { powder }\end{array}$ & 146 & 12.4 & 65 & 9.2 & 19 & 5.6 & 132 & 7.8 & 59 & 7.5 & 23 & 5.5 & 444 & 8.7 \\
\hline GHB or GBL & 136 & 11.5 & 46 & 6.5 & 9 & 2.7 & 119 & 7.1 & 58 & 7.4 & 8 & 1.9 & 373 & 7.3 \\
\hline Crystal methamphetamine & 80 & 6.8 & 43 & 6.1 & 16 & 4.7 & 129 & 7.6 & 29 & 3.7 & 13 & 3.1 & 310 & 6.1 \\
\hline \multicolumn{15}{|l|}{ Ever injected any drugs ${ }^{f}$} \\
\hline No, never & 1,139 & 96.0 & 679 & 95.8 & 328 & 96.8 & 1,640 & 96.6 & 770 & 98.1 & 413 & 96.3 & 4,969 & 96.5 \\
\hline Yes & 48 & 4.0 & 30 & 4.2 & 11 & 3.2 & 58 & 3.4 & 15 & 1.9 & 16 & 3.7 & 178 & 3.5 \\
\hline
\end{tabular}

Abbreviations: GBL, gamma-Butyrolactone; GHB, Gamma-hydroxybutyrate

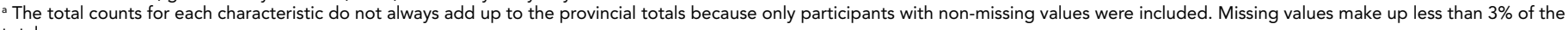
totals

${ }^{b}$ Because someone knew or presumed the participant was attracted to men

c PHQ-4 measurement (13)

${ }^{d}$ CAGE 4-item Indicator (14)

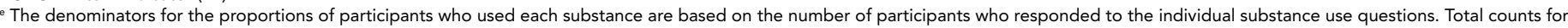
each substance ranged from 5,113 to 5,121 . The proportions for substances used do not add up to $100 \%$ as they were not mutually exclusive; participants could report the use of more than one substance

Other than anabolic steroids or prescribed medicine 
time of the survey, cocaine (14.0\%) and ecstasy pills (8.9\%) (Table 2). Substances used in the previous year by less than $5 \%$ of participants overall were as follows: amphetamine $(4.4 \%)$, ketamine $(4.0 \%)$, LSD (3.9\%), synthetic cannabinoids $(2.1 \%)$, crack cocaine $(1.5 \%)$, heroin $(0.9 \%)$, mephedrone $(0.4 \%)$ and synthetic stimulants other than mephedrone (0.5\%). Only $3.5 \%$ of participants reported ever injecting drugs.

\section{Knowledge and use of PEP and PrEP}

Most participants had heard of PrEP (86.4\%); a slightly smaller proportion had heard of PEP (74.3\%), and $8.4 \%$ of all the participants, regardless of HIV status, had taken or were currently taking PrEP (Table 3). Of HIV-negative or untested men, $51.7 \%$ reported being likely to use PrEP if it was available and affordable.

Table 3: Awareness and use of PEP and PrEP, and healthcare provider knowledge of sexual attraction to men of Canadian participants in the EMIS-2017 $(\mathrm{N}=5,165)$

\begin{tabular}{|c|c|c|c|c|c|c|c|c|c|c|c|c|c|c|}
\hline \multirow[t]{2}{*}{ Characteristics } & \multicolumn{2}{|c|}{$\begin{array}{l}\text { British } \\
\text { Columbia }+ \\
\text { Yukon } \\
(n=1,191)^{a}\end{array}$} & \multicolumn{2}{|c|}{$\begin{array}{l}\text { Alberta + } \\
\text { Northwest } \\
\text { Territories } \\
(n=710)^{a}\end{array}$} & \multicolumn{2}{|c|}{$\begin{array}{c}\text { Saskatchewan } \\
+ \\
\text { Manitoba } \\
(n=339)^{a}\end{array}$} & \multicolumn{2}{|c|}{$\begin{array}{c}\text { Ontario } \\
+ \\
\text { Nunavut } \\
(n=1,707)^{a}\end{array}$} & \multicolumn{2}{|c|}{$\begin{array}{l}\text { Quebec } \\
(n=789)^{a}\end{array}$} & \multicolumn{2}{|c|}{$\begin{array}{c}\text { Atlantic } \\
\text { Provinces } \\
(n=429)^{a}\end{array}$} & \multicolumn{2}{|c|}{$\begin{array}{c}\text { Total } \\
(\mathrm{N}=5,165)^{\mathrm{a}}\end{array}$} \\
\hline & $n$ & $\%$ & $n$ & $\%$ & $\mathbf{n}$ & $\%$ & $n$ & $\%$ & $\mathbf{n}$ & $\%$ & $n$ & $\%$ & $n$ & $\%$ \\
\hline \multicolumn{15}{|l|}{ Heard of PEP } \\
\hline No & 157 & 13.4 & 175 & 24.8 & 76 & 22.6 & 265 & 15.7 & 167 & 21.3 & 117 & 27.8 & 957 & 18.7 \\
\hline Yes & 958 & 81.5 & 465 & 65.9 & 222 & 66.1 & 1,323 & 78.6 & 560 & 71.5 & 268 & 63.7 & 3,796 & 74.3 \\
\hline Not sure & 61 & 5.2 & 66 & 9.3 & 38 & 11.3 & 96 & 5.7 & 56 & 7.2 & 36 & 8.6 & 353 & 6.9 \\
\hline \multicolumn{15}{|l|}{ Ever taken PEP ${ }^{b}$} \\
\hline No, have not tried to get it & 923 & 89.4 & 634 & 95.5 & 304 & 94.4 & 1,395 & 91.8 & 629 & 87.1 & 386 & 95.5 & 4,271 & 91.6 \\
\hline No, tried, but could not get it & 20 & 1.9 & 12 & 1.8 & 8 & 2.5 & 29 & 1.9 & 14 & 1.9 & 9 & 2.2 & 92 & 2.0 \\
\hline $\begin{array}{l}\text { No, had the opportunity but } \\
\text { decided not to }\end{array}$ & 14 & 1.4 & 5 & 0.8 & 4 & 1.2 & 19 & 1.3 & 13 & 1.8 & 1 & 0.2 & 56 & 1.2 \\
\hline Yes, one or more courses & 76 & 7.4 & 13 & 2.0 & 6 & 1.9 & 77 & 5.1 & 66 & 9.1 & 8 & 2.0 & 246 & 5.3 \\
\hline \multicolumn{15}{|c|}{ Confidence in ability to access PEPb } \\
\hline Very confident & 322 & 31.2 & 145 & 21.9 & 71 & 22.0 & 470 & 30.9 & 262 & 36.2 & 69 & 17.0 & 1,339 & 28.7 \\
\hline Quite confident & 268 & 25.9 & 149 & 22.5 & 66 & 20.5 & 344 & 22.6 & 231 & 31.9 & 70 & 17.2 & 1,128 & 24.1 \\
\hline A little confident & 173 & 16.7 & 126 & 19.0 & 64 & 19.9 & 287 & 18.8 & 98 & 13.5 & 93 & 22.9 & 841 & 18.0 \\
\hline Not at all confident & 118 & 11.4 & 107 & 16.1 & 56 & 17.4 & 192 & 12.6 & 49 & 6.8 & 79 & 19.5 & 601 & 12.9 \\
\hline I don't know & 152 & 14.7 & 136 & 20.5 & 65 & 20.2 & 230 & 15.1 & 84 & 11.6 & 95 & 23.4 & 762 & 16.3 \\
\hline \multicolumn{15}{|l|}{ Heard of PrEP } \\
\hline No & 88 & 7.5 & 96 & 13.7 & 53 & 15.8 & 146 & 8.7 & 93 & 11.9 & 79 & 18.8 & 555 & 10.9 \\
\hline Yes & 1,063 & 90.7 & 576 & 82.1 & 265 & 79.1 & 1,506 & 89.3 & 669 & 85.7 & 326 & 77.6 & 4,405 & 86.4 \\
\hline Not sure & 21 & 1.8 & 30 & 4.3 & 17 & 5.1 & 34 & 2.0 & 19 & 2.4 & 15 & 3.6 & 136 & 2.7 \\
\hline \multicolumn{15}{|l|}{ Ever taken PrEP } \\
\hline No & 1,099 & 92.4 & 674 & 95.5 & 325 & 95.9 & 1,532 & 90.3 & 664 & 84.7 & 415 & 97.6 & 4,701 & 91.6 \\
\hline Yes & 90 & 7.6 & 32 & 4.6 & 14 & 4.1 & 165 & 9.7 & 120 & 15.3 & 10 & 2.4 & 431 & 8.4 \\
\hline \multicolumn{15}{|c|}{ Likelihood of using PrEP if available and affordable ${ }^{c}$} \\
\hline Very unlikely & 117 & 11.3 & 46 & 6.9 & 30 & 9.3 & 143 & 9.4 & 88 & 12.2 & 36 & 8.9 & 460 & 9.9 \\
\hline Quite unlikely & 111 & 10.7 & 50 & 7.6 & 39 & 12.1 & 134 & 8.8 & 99 & 13.7 & 32 & 7.9 & 465 & 10.0 \\
\hline Not sure & 277 & 26.8 & 185 & 27.9 & 79 & 24.5 & 440 & 29.0 & 200 & 27.7 & 143 & 35.5 & 1,324 & 28.4 \\
\hline Quite likely & 185 & 17.9 & 156 & 23.6 & 74 & 23.0 & 319 & 21.0 & 145 & 20.1 & 88 & 21.8 & 967 & 20.7 \\
\hline Very likely & 343 & 33.2 & 225 & 34.0 & 100 & 31.1 & 483 & 31.8 & 190 & 26.3 & 104 & 25.8 & 1,445 & 31.0 \\
\hline
\end{tabular}




\section{Sexual practices}

Over half of participants (62.1\%) always or almost always had sober sex in the previous 12 months (Table 4). One-fifth (21.5\%) of the participants reported participation in chemsex, and $5.8 \%$ participated in chemsex in the past six months.
With regard to sexual practices, $62.1 \%$ of the participants reported any anal intercourse with casual partners; of these men, $73.6 \%$ were inconsistent in their condom use in the previous year (ranging from one to multiple condomless acts).

Table 4: Sexual practices of Canadian participants in the EMIS-2017 $(N=5,165)$

\begin{tabular}{|c|c|c|c|c|c|c|c|c|c|c|c|c|c|c|}
\hline \multirow[t]{2}{*}{ Characteristics } & \multicolumn{2}{|c|}{$\begin{array}{c}\text { British Columbia } \\
+ \text { Yukon } \\
(n=1,191)^{\mathrm{a}}\end{array}$} & \multicolumn{2}{|c|}{$\begin{array}{c}\text { Alberta + } \\
\text { Northwest } \\
\text { Territories } \\
(\mathrm{n}=710)^{\mathrm{a}}\end{array}$} & \multicolumn{2}{|c|}{$\begin{array}{c}\text { Saskatchewan + } \\
\text { Manitoba } \\
(n=339)^{a}\end{array}$} & \multicolumn{2}{|c|}{$\begin{array}{c}\text { Ontario } \\
+ \\
\text { Nunavut } \\
(n=1,707)^{a}\end{array}$} & \multicolumn{2}{|c|}{$\begin{array}{l}\text { Quebec } \\
(n=789)^{a}\end{array}$} & \multicolumn{2}{|c|}{$\begin{array}{c}\text { Atlantic Provinces } \\
(n=429)\end{array}$} & \multicolumn{2}{|c|}{$\begin{array}{c}\text { Total } \\
(\mathrm{N}=5,165)^{\mathrm{a}}\end{array}$} \\
\hline & $\mathbf{n}$ & $\%$ & \begin{tabular}{l|l}
$\mathbf{n}$ & \\
\end{tabular} & $\%$ & $\mathbf{n}$ & $\%$ & \begin{tabular}{l|l}
$\mathbf{n}$ & \\
\end{tabular} & $\%$ & \begin{tabular}{l|l}
$\mathbf{n}$ & \\
\end{tabular} & $\%$ & $\mathbf{n}$ & $\%$ & \begin{tabular}{l|l}
$\mathbf{n}$ &
\end{tabular} & $\%$ \\
\hline \multicolumn{15}{|c|}{ Nonsober sex (last 12 months) ${ }^{b}$} \\
\hline None of it & 362 & 33.1 & 252 & 41.0 & 125 & 41.7 & 533 & 35.0 & 253 & 35.8 & 160 & 44.3 & 1,685 & 36.6 \\
\hline Almost none of it & 278 & 25.4 & 174 & 28.3 & 81 & 27.0 & 380 & 25.0 & 171 & 24.2 & 87 & 24.1 & 1,171 & 25.5 \\
\hline Less than half & 141 & 12.9 & 71 & 11.6 & 26 & 8.7 & 209 & 13.7 & 92 & 13.0 & 42 & 11.6 & 581 & 12.6 \\
\hline About half & 115 & 10.5 & 42 & 6.8 & 21 & 7.0 & 122 & 8.0 & 67 & 9.5 & 24 & 6.6 & 391 & 8.5 \\
\hline More than half & 74 & 6.8 & 27 & 4.4 & 9 & 3.0 & 111 & 7.3 & 49 & 6.9 & 16 & 4.4 & 286 & 6.2 \\
\hline Almost all of it & 97 & 8.9 & 31 & 5.0 & 31 & 10.3 & 121 & 8.0 & 58 & 8.2 & 23 & 6.4 & 361 & 7.8 \\
\hline All of it & 28 & 2.6 & 17 & 2.8 & 7 & 2.3 & 46 & 3.0 & 17 & 2.4 & 9 & 2.5 & 124 & 2.7 \\
\hline \multicolumn{15}{|l|}{ Recency of chemsex ${ }^{c}$} \\
\hline Within six months & 95 & 8.1 & 26 & 3.7 & 11 & 3.3 & 105 & 6.2 & 47 & 6.0 & 14 & 3.3 & 298 & 5.8 \\
\hline Six months to a year & 76 & 6.5 & 56 & 7.9 & 14 & 4.1 & 127 & 7.5 & 50 & 6.4 & 20 & 4.7 & 343 & 6.7 \\
\hline $1-5$ years ago & 50 & 4.2 & 37 & 5.2 & 10 & 3.0 & 62 & 3.7 & 31 & 3.9 & 12 & 2.8 & 202 & 3.9 \\
\hline $5+$ years ago & 77 & 6.5 & 31 & 4.4 & 8 & 2.4 & 84 & 5.0 & 40 & 5.1 & 20 & 4.7 & 260 & 5.1 \\
\hline Never & 879 & 74.7 & 558 & 78.8 & 295 & 87.3 & 1,311 & 77.6 & 617 & 78.6 & 357 & 84.4 & 4,017 & 78.5 \\
\hline \multicolumn{15}{|c|}{ Anal intercourse with casual partners (participants of any HIV status) ${ }^{d}$} \\
\hline No anal intercourse & 420 & 36 & 258 & 36.7 & 125 & 37.3 & 632 & 37.8 & 308 & 39.6 & 181 & 42.9 & 1,924 & 37.9 \\
\hline Any anal intercourse & 748 & 64 & 444 & 63.3 & 210 & 62.7 & 1,038 & 62.2 & 470 & 60.4 & 241 & 57.1 & 3,151 & 62.1 \\
\hline \multicolumn{15}{|c|}{ Consistent condom use with casual partners (participants of any HIV status) } \\
\hline Non-consistent condom use & 561 & 75.0 & 327 & 73.6 & 160 & 76.2 & 765 & 73.8 & 320 & 68.2 & 185 & 76.8 & 2,318 & 73.6 \\
\hline Consistent condom use & 187 & 25.0 & 117 & 26.4 & 50 & 23.8 & 272 & 26.2 & 149 & 31.8 & 56 & 23.2 & 831 & 26.4 \\
\hline \multicolumn{15}{|c|}{ Any CAI by HIV-negative men, on PrEP ${ }^{f}$} \\
\hline No CAl & - & - & - & - & - & - & - & - & - & - & - & - & 30 & 8.7 \\
\hline Any CAl & - & - & - & - & - & - & - & - & - & - & - & - & 314 & 91.3 \\
\hline \multicolumn{15}{|c|}{ Any CAl among HIV-negative or HIV-unknown men not on PrEPg } \\
\hline No CAl & 180 & 26.5 & 114 & 27.4 & 50 & 25.1 & 265 & 29.5 & 134 & 36.1 & 54 & 23.4 & 797 & 28.5 \\
\hline Any CAl & 498 & 73.5 & 302 & 72.6 & 149 & 74.9 & 632 & 70.5 & 237 & 63.9 & 177 & 76.6 & 1,995 & 71.5 \\
\hline \multicolumn{15}{|c|}{ Any CAI by HIV-positive men, on treatment, undetectable viral load ${ }^{\text {h }}$} \\
\hline No CAl & - & - & - & - & - & - & - & - & - & - & - & - & 22 & 7.3 \\
\hline Any CAI & - & - & - & - & - & - & - & - & - & - & - & - & 278 & 92.7 \\
\hline \multicolumn{15}{|c|}{ Any CAl among HIV-positive men not on treatment and detectable viral load } \\
\hline No CAl & - & - & - & - & - & - & - & - & - & - & - & - & 12 & 85.7 \\
\hline Any CAl & - & - & - & - & - & - & - & - & - & - & - & - & 2 & 14.3 \\
\hline \multicolumn{15}{|l|}{ Paid a man to have sex } \\
\hline Within 12 months & 57 & 4.9 & 33 & 4.8 & 24 & 7.4 & 108 & 6.6 & 51 & 6.8 & 14 & 3.5 & 287 & 5.8 \\
\hline $1-5$ years & 61 & 5.3 & 27 & 4.0 & 15 & 4.6 & 89 & 5.4 & 34 & 4.5 & 16 & 4.0 & 242 & 4.9 \\
\hline $5+$ years & 78 & 6.7 & 31 & 4.6 & 19 & 5.9 & 113 & 6.9 & 38 & 5.1 & 18 & 4.4 & 297 & 6.0 \\
\hline Never & 961 & 83.1 & 590 & 86.6 & 266 & 82.1 & 1,333 & 81.1 & 626 & 83.6 & 357 & 88.1 & 4,133 & 83.3 \\
\hline \multicolumn{15}{|l|}{ Paid by a man to have sex } \\
\hline Within 12 months & 40 & 3.5 & 30 & 4.4 & 7 & 2.2 & 67 & 4.1 & 36 & 4.8 & 20 & 4.9 & 200 & 4.0 \\
\hline $1-5$ years ago & 44 & 3.8 & 22 & 3.2 & 12 & 3.7 & 72 & 4.4 & 44 & 5.9 & 17 & 4.2 & 211 & 4.3 \\
\hline $5+$ years ago & 114 & 9.9 & 43 & 6.3 & 24 & 7.4 & 153 & 9.3 & 33 & 4.4 & 25 & 6.2 & 392 & 7.9 \\
\hline Never & 959 & 82.9 & 586 & 86.0 & 281 & 86.7 & 1,353 & 82.2 & 637 & 84.9 & 344 & 84.7 & 4,160 & 83.8 \\
\hline
\end{tabular}

Abbreviations: CAl, condomless anal intercourse; PrEP, preexposure prophylaxis; -, numbers were suppressed due to small cell sizes

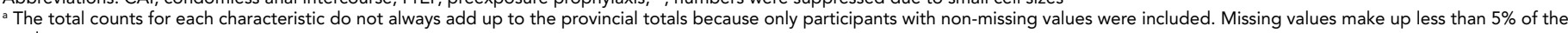
totals

${ }^{b}$ Among men who have had sex with a man in last 12 months $(\mathrm{N}=4,599)$

' Used stimulant drugs to make sex more intense or last longer. Note: The stimulant drugs include ecstasy/MDMA, cocaine, amphetamine (speed), crystal methamphetamine (Tina, Pervitin),

mephedrone and ketamine

Among men of any HIV status who had one or more casual partners in the last 12 months $(N=5,075)$

Among men of any HIV status who had anal intercourse with one or more casual partners in the last 12 months $(N=3,149)$

${ }^{f}$ Among HIV-negative men on PrEP who had anal intercourse with one or more casual partners in last 12 months $(\mathrm{N}=344)$

9 Among HIV-negative or untested men not on PrEP who had anal intercourse with one or more casual partners in last 12 months ( $\mathrm{N}=2,792$ )

Among HIV-positive men on treatment and undetectable viral load who had anal intercourse with one or more casual partners in last 12 months ( $N=300$ )

Among HIV-positive men not on treatment and with a detectable viral load who had anal intercourse with one or more casual partners in last 12 months ( $\mathrm{N}=21$ ) 
Of the HIV-negative men using PrEP who had anal intercourse with one or more casual partners in the past year $(n=344), 91.3 \%$ participated in any CAI with casual partners in the past year. Of those who were HIV-negative or did not know their status, who had anal intercourse with one or more casual partners in the past year and were currently not using $\operatorname{PrEP}(n=2,792)$, $71.5 \%$ participated in any CAl with casual partners in the past year. Of the HIV-positive participants who had anal intercourse with one or more casual partners in the past year and who had an undetectable viral load $(n=300), 92.7 \%$ participated in any CAl with casual partners in the past year. Of the men who were HIV-positive who had anal intercourse with one or more casual partners in the past year, were not taking antiretroviral therapy and had a detectable viral load $(n=14), 14.3 \%$ had engaged in CAl with casual partners in the past year.

In the past year, $5.8 \%$ of participants bought sex and $4.0 \%$ sold sex with male partners.

\section{STBBI testing and diagnosis}

Although almost two-thirds of participants (62.5\%) had tested for HIV in the previous year, only one-quarter (24.9\%) reported full STI screening in the last 12 months (Table 5). This proportion was higher (37.7\%) among participants who engaged in any CAl in the previous year.

Most of the participants (90\%) who had been tested for STIs other than HIV infection in the previous year stated that their healthcare provider knew they had sex with other men.

With regard to bloodborne infections, $1.5 \%$ of participants were ever diagnosed with hepatitis $C$ and $9 \%$ reporting being diagnosed with HIV infection. Of those with an HIV diagnosis, $99.1 \%$ of the HIV-positive participants reported current use of antiretroviral therapies; of those, $96.7 \%$ reported an undetectable viral load. For other STBBIs, $3.2 \%$ of the participants had received a syphilis diagnosis within the previous year. The proportions of participants diagnosed in the previous year with gonorrhea and chlamydia (including lymphogranuloma venereum) were $7.1 \%$ and $6.5 \%$, respectively.

Table 5: Sexually transmitted and bloodborne infection testing and diagnoses of Canadian participants in the EMIS-2017 $(\mathrm{N}=5,165)$

\begin{tabular}{|c|c|c|c|c|c|c|c|c|c|c|c|c|c|c|}
\hline \multirow[t]{2}{*}{ Characteristics } & \multicolumn{2}{|c|}{$\begin{array}{c}\text { British } \\
\text { Columbia }+ \\
\text { Yukon } \\
(n=1,191)^{\mathrm{a}}\end{array}$} & \multicolumn{2}{|c|}{$\begin{array}{c}\text { Alberta + } \\
\text { Northwest } \\
\text { Territories } \\
(n=710)^{\mathrm{a}}\end{array}$} & \multicolumn{2}{|c|}{$\begin{array}{c}\text { Saskatchewan } \\
+ \\
\text { Manitoba } \\
(n=339)^{\mathrm{a}}\end{array}$} & \multicolumn{2}{|c|}{$\begin{array}{c}\text { Ontario } \\
+ \\
\text { Nunavut } \\
(n=1,707)^{a}\end{array}$} & \multicolumn{2}{|c|}{$\begin{array}{l}\text { Quebec } \\
(n=789)^{a}\end{array}$} & \multicolumn{2}{|c|}{$\begin{array}{c}\text { Atlantic } \\
\text { Provinces } \\
(n=429)^{a}\end{array}$} & \multicolumn{2}{|c|}{$\begin{array}{c}\text { Total } \\
(\mathrm{N}=5,165)^{\mathrm{a}}\end{array}$} \\
\hline & $\mathbf{n}$ & $\%$ & $n$ & $\%$ & $n$ & $\%$ & $\mathbf{n}$ & $\%$ & $\mathrm{n}$ & $\%$ & $\mathrm{n}$ & $\%$ & $\mathbf{n}$ & $\%$ \\
\hline \multicolumn{15}{|c|}{ Received full STI screen in last 12 months ${ }^{b}$} \\
\hline No & 698 & 66.7 & 480 & 71.6 & 246 & 76.4 & 1,270 & 82.5 & 473 & 64.9 & 377 & 92.0 & 3,544 & 75.1 \\
\hline Yes & 348 & 33.3 & 190 & 28.4 & 76 & 23.6 & 269 & 17.5 & 256 & 35.1 & 33 & 8.0 & 1,172 & 24.9 \\
\hline \multicolumn{15}{|c|}{ Healthcare provider's knowledge of sex with men } \\
\hline Definitely/Probably knew & 718 & 91.8 & 358 & 86.1 & 153 & 86.4 & 883 & 91.3 & 451 & 93.8 & 155 & 78.7 & 2,718 & 90.0 \\
\hline $\begin{array}{l}\text { Did not know or Don't know if they } \\
\text { knew }\end{array}$ & 64 & 8.2 & 58 & 13.9 & 24 & 13.6 & 84 & 8.7 & 30 & 6.2 & 42 & 21.3 & 302 & 10.0 \\
\hline \multicolumn{15}{|l|}{ HIV testing history ${ }^{d}$} \\
\hline Within six months & 213 & 20.6 & 94 & 14.2 & 39 & 12.1 & 224 & 14.7 & 131 & 18.1 & 26 & 6.4 & 727 & 15.5 \\
\hline Six months to a year & 507 & 49.0 & 311 & 46.8 & 150 & 46.6 & 731 & 47.9 & 327 & 45.2 & 173 & 42.6 & 2,199 & 47.0 \\
\hline $1-5$ years & 151 & 14.6 & 91 & 13.7 & 36 & 11.2 & 219 & 14.4 & 103 & 14.2 & 67 & 16.5 & 667 & 14.3 \\
\hline $5+$ years & 45 & 4.4 & 26 & 3.9 & 22 & 6.8 & 83 & 5.4 & 28 & 3.9 & 25 & 6.2 & 229 & 4.9 \\
\hline Never & 118 & 11.4 & 142 & 21.4 & 75 & 23.3 & 269 & 17.6 & 135 & 18.6 & 115 & 28.3 & 854 & 18.3 \\
\hline \multicolumn{15}{|l|}{ Last syphilis diagnosis } \\
\hline Within 12 months & 50 & 4.2 & 21 & 3.0 & 8 & 2.4 & 53 & 3.2 & 25 & 3.2 & 5 & 1.2 & 162 & 3.2 \\
\hline $1-5$ years ago & 71 & 6.0 & 24 & 3.4 & 16 & 4.8 & 93 & 5.5 & 47 & 6.1 & 20 & 4.7 & 271 & 5.3 \\
\hline $5+$ years & 44 & 3.7 & 22 & 3.1 & 9 & 2.7 & 84 & 5.0 & 19 & 2.5 & 14 & 3.3 & 192 & 3.8 \\
\hline Never & 1,015 & 86.0 & 635 & 90.5 & 301 & 90.1 & 1,447 & 86.3 & 684 & 88.3 & 387 & 90.8 & 4,469 & 87.7 \\
\hline \multicolumn{15}{|l|}{ Last gonorrhea diagnosis } \\
\hline Within 12 months & 110 & 9.3 & 43 & 6.1 & 19 & 5.7 & 99 & 5.9 & 77 & 10.0 & 11 & 2.6 & 359 & 7.1 \\
\hline $1-5$ years ago & 147 & 12.5 & 60 & 8.5 & 22 & 6.6 & 135 & 8.0 & 72 & 9.3 & 17 & 4.0 & 453 & 8.9 \\
\hline $5+$ years ago & 131 & 11.1 & 60 & 8.5 & 22 & 6.6 & 180 & 10.7 & 65 & 8.4 & 32 & 7.5 & 490 & 9.6 \\
\hline Never & 790 & 67.1 & 540 & 76.8 & 268 & 81.0 & 1,268 & 75.4 & 559 & 72.3 & 365 & 85.9 & 3,790 & 74.4 \\
\hline \multicolumn{15}{|l|}{ Last chlamydia or LGV diagnosis } \\
\hline Within 12 months & 105 & 9.0 & 35 & 5.1 & 14 & 4.3 & 106 & 6.4 & 60 & 7.8 & 7 & 1.7 & 327 & 6.5 \\
\hline $1-5$ years ago & 109 & 9.3 & 57 & 8.2 & 26 & 7.9 & 140 & 8.4 & 67 & 8.7 & 18 & 4.3 & 417 & 8.2 \\
\hline $5+$ years ago & 94 & 8.0 & 43 & 6.2 & 24 & 7.3 & 127 & 7.6 & 42 & 5.4 & 21 & 5.0 & 351 & 6.9 \\
\hline Never & 865 & 73.7 & 558 & 80.5 & 265 & 80.5 & 1,293 & 77.6 & 604 & 78.1 & 375 & 89.1 & 3,960 & 78.3 \\
\hline
\end{tabular}


Table 5: Sexually transmitted and bloodborne infection testing and diagnoses of Canadian participants in the EMIS-2017 $(\mathrm{N}=5,165)$ (continued)

\begin{tabular}{|c|c|c|c|c|c|c|c|c|c|c|c|c|c|c|}
\hline \multirow[t]{2}{*}{ Characteristics } & \multicolumn{2}{|c|}{$\begin{array}{c}\text { British } \\
\text { Columbia }+ \\
\text { Yukon } \\
(n=1,191)^{a}\end{array}$} & \multicolumn{2}{|c|}{$\begin{array}{c}\text { Alberta + } \\
\text { Northwest } \\
\text { Territories } \\
(n=710)^{a}\end{array}$} & \multicolumn{2}{|c|}{$\begin{array}{c}\text { Saskatchewan } \\
+ \\
\text { Manitoba } \\
(n=339)^{a}\end{array}$} & \multicolumn{2}{|c|}{$\begin{array}{c}\text { Ontario } \\
+ \\
\text { Nunavut } \\
(n=1,707)^{a}\end{array}$} & \multicolumn{2}{|c|}{$\begin{array}{l}\text { Quebec } \\
(n=789)^{a}\end{array}$} & \multicolumn{2}{|c|}{$\begin{array}{l}\text { Atlantic } \\
\text { Provinces } \\
(n=429)^{a}\end{array}$} & \multicolumn{2}{|c|}{$\begin{array}{c}\text { Total } \\
(\mathrm{N}=5,165)^{\mathrm{a}}\end{array}$} \\
\hline & $\mathbf{n}$ & $\%$ & $n$ & $\%$ & $n$ & $\%$ & $\mathrm{n}$ & $\%$ & $\mathbf{n}$ & $\%$ & $n$ & $\%$ & $\mathbf{n}$ & $\%$ \\
\hline \multicolumn{15}{|c|}{ Ever diagnosed with hepatitis C } \\
\hline No & 1,135 & 95.3 & 672 & 94.9 & 331 & 97.9 & 1,648 & 96.8 & 765 & 97.2 & 408 & 95.8 & 4,959 & 96.3 \\
\hline Yes & 26 & 2.2 & 13 & 1.8 & 0 & 0.0 & 23 & 1.4 & 11 & 1.4 & 3 & 0.7 & 76 & 1.5 \\
\hline I don't know & 30 & 2.5 & 23 & 3.2 & 7 & 2.1 & 31 & 1.8 & 11 & 1.4 & 15 & 3.5 & 117 & 2.3 \\
\hline \multicolumn{15}{|l|}{ Ever diagnosed with HIV } \\
\hline No & 1,034 & 87.4 & 664 & 94.1 & 322 & 95.0 & 1,526 & 89.8 & 724 & 92.0 & 406 & 95.5 & 4,676 & 91.0 \\
\hline Yes & 149 & 12.6 & 42 & 5.9 & 17 & 5.0 & 174 & 10.2 & 63 & 8.0 & 19 & 4.5 & 464 & 9.0 \\
\hline \multicolumn{15}{|l|}{ Currently taking ART } \\
\hline No & - & - & - & - & - & - & - & - & - & - & - & - & 4 & 0.9 \\
\hline Yes & - & - & - & - & - & - & - & - & - & - & - & - & 436 & 99.1 \\
\hline \multicolumn{15}{|l|}{ Detectable at last viral load } \\
\hline Undetectable & - & - & - & - & - & - & - & - & - & - & - & - & 440 & 96.7 \\
\hline Detectable & - & - & - & - & - & - & - & - & - & - & - & - & 15 & 3.3 \\
\hline \multicolumn{15}{|c|}{ 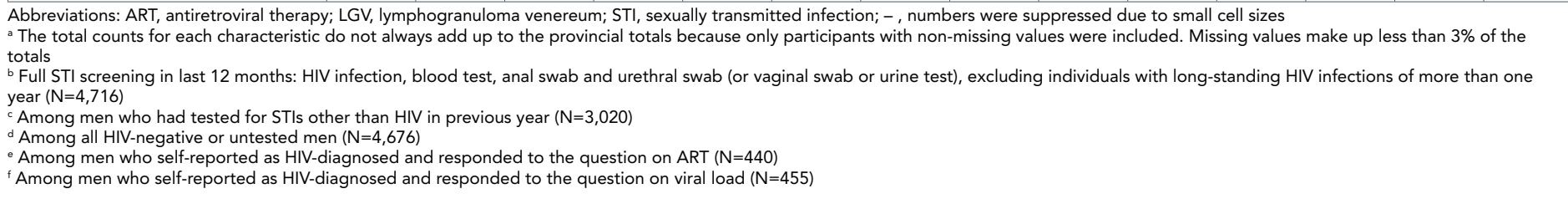 } \\
\hline
\end{tabular}

\section{Discussion}

EMIS-2017 identified that Canadian participants experienced high levels of intimidation, as well as verbal abuse and physical violence related to their attraction to other men. Moderate to severe anxiety or depression were present in almost $25 \%$ of participants. Substance use was high and, for the first time, there are national data showing that over $20 \%$ of participants engaged in chemsex. There was a significant gap between the proportion of participants who reported using PrEP and the proportion who reported they would use PrEP if it was readily available: $8.4 \%$ versus $51.7 \%$. Although virtually all HIV-diagnosed respondents were undergoing treatment and had undetectable viral loads, less than $25 \%$ stated that they had received full STI testing in the previous year.

\section{Strengths and limitations}

The strength of the Canadian EMIS-2017 survey was the use of a global, validated questionnaire that will facilitate cross-country comparisons, a large sample size and participation from all regions of Canada.

A number of limitations need to be considered when interpreting the findings. Since the EMIS-2017 was based on self-reported data and included sensitive topics such as sexual practices and substance use, some degree of underreporting of higher-risk behaviours may have occurred. However, any underreporting was likely limited given the self-administered nature of the survey. EMIS-2017 made use of non-probability sampling methods, including the use of social and sexual networking mobile applications for recruitment, and, as a result, it more likely represents sexually active nonmonogamous gbMSM. However, this is a main target population for both behavioural surveys and public health interventions.

\section{Implications}

Many of the findings are consistent with previous studies. For example, a Canadian survey of gbMSM found a higher risk of suicidal ideation and related behaviour among gbMSM than among heterosexual men (17). A review of the international literature found a higher prevalence of substance use among gbMSM than heterosexual men (18). The reported use of chemsex $(21.5 \%)$ in the Canadian portion of the EMIS-2017 was higher than found by regional Canadian studies $(6 \%$ and $18 \%)(19,20)$ and may reflect regional variation. Other studies have also found that mental health challenges and substance use are associated with gbMSM engaging in higher-risk sexual practices (21-23). The tendency of these issues to coincide has been conceptualized as a syndemic, defined as co-occurring epidemics that results in a higher disease burden in marginalized populations (24). Previous studies have indicated that in some cities between $10.5 \%$ and $12.5 \%$ of gbMSM are using PrEP (25), and that $50 \%$ to $60 \%$ of gbMSM are interested and willing to use $\operatorname{PrEP}(26,27)$, suggesting affordability and accessibility are barriers $(28,29)$. A low proportion of participants reporting full STI testing have been found in other surveys of gbMSM in Canada (30) and internationally $(31,32)$. The finding that almost all participants diagnosed with HIV infection were undergoing 
treatment and had undetectable viral loads is similar to recent regional surveys of gbMSM $(33,34)$.

\section{Next steps}

The EMIS-2017 findings point to the need for implementation research to determine best practices to address the high levels of discrimination, poor mental health and substance use harms that gbMSM experience. The EMIS-2017 study also provides useful baseline data on PrEP. In light of changes to provincial formularies and recent guidelines on PrEP prescribing, we anticipate an uptake of this effective prevention technology. Further research would be useful in determining the role of PrEP on sexual risk practices and on the subsequent rates of infection with HIV and other STBBls. As this survey likely captured a specific and important subgroup of gbMSM regarding STBBls, triangulating these data with information generated from future surveys using alternative sampling methods would lead to a more comprehensive understanding of this population as a whole.

\section{Conclusion}

gbMSM in Canada experienced high levels of stigma,

discrimination, mental health problems, and substance use.

Furthermore, a low prevalence of condom use was found among them. The gap between the proportion of men who were interested in PrEP and those who actually used it is significant and comprehensive STBBI testing was low. These findings can inform public health action and provide a baseline to examine the impact of current and new interventions.

\section{Authors' statement}

NB - Conceptualization, data curation, formal analysis, writing (original draft and review and editing)

DMP - Funding acquisition, project administration, conceptualization, data curation, formal analysis, writing - review and editing

NJL - Conceptualization, formal analysis, writing (review and editing)

MB - Conceptualization, formal analysis, writing (review and editing)

DJB - Conceptualization, formal analysis, writing (review and editing)

$\mathrm{TAH}$ - Conceptualization, formal analysis, writing (review and editing)

BA - Conceptualization, formal analysis, writing (review and editing)

\section{Conflict of interest}

None.

\section{Acknowledgements}

European Men-who-have-sex-with-men Internet Survey (EMIS-2017) was undertaken by Sigma Research at the London School of Hygiene and Tropical Medicine in association with the Robert Koch Institute in Berlin. The EMIS core team is made up of F Hickson, D Reid, AJ Schmidt and P Weatherburn at the London School of Hygiene and Tropical Medicine and U Marcus and SB Schink at the Robert Koch Institute. We especially thank AJ Schmidt for his continued and strong support of Canadian participation in EMIS-2017.

We thank all of the men who took part in EMIS-2017 and our nongovernmental organization (NGO) partners, particularly the Health Initiative for Men, Rézo, the Gay Men's Sexual Health Alliance of Ontario and Canadian AIDS Treatment Information Exchange (CATIE), who recruited participants through activities on Facebook and/or other social media and by placing EMIS banners on their websites. We thank all our partners for being part of something huge. We also would like to thank $C$ Archibald for his advice prior to and throughout data collection and for his review of the manuscript.

\section{Funding}

As part of European Surveys and Trainings to Improve MSM Community Health project, EMIS-2017 was carried out under the service contract 20157101 with the Consumers, Health, Agriculture and Food Executive Agency (Chafea), acting under powers delegated by the Commission of the European Union. Other financial contributions came from the Public Health Agency of Canada (PHAC) for recruitment in Canada. NJL is supported by a Scholar Award from the Michael Smith Foundation for Health Research (\#16863). TAH is supported by an Endgame Leader Chair Award from the Ontario HIV Treatment Network. Promotion of the survey and recruitment of Canadian participants in EMIS-2017 was funded by the PHAC.

\section{References}

1. The ESTICOM Network. EMIS-2017 - The European MenWho-Have-Sex-With-Men Internet Survey. Key finding from 50 countries. Stockholmn (SE): European Centre for Disease Prevention and Control; 2019 (Accessed 2019-09-04). http://www.emis2017.eu

2. Public Health Agency of Canada. Summary: estimates of HIV incidence, prevalence and Canada's progress on meeting the 90-90-90 HIV targets, 2016. Ottawa (ON): PHAC; 2018. https://www.canada.ca/content/dam/phac-aspc/documents/ services/publications/diseases-conditions/summary-estimate s-hiv-incidence-prevalence-canadas-progress-90-90-90/ pub-eng.pdf 
3. Choudhri Y, Miller J, Sandhu J, Leon A, Aho J. Infectious and congenital syphilis in Canada, 2010-2015. Can Commun Dis Rep 2018 Feb;44(2):43-8. DOI PubMed

4. Choudhri Y, Miller J, Sandhu J, Leon A, Aho J. Gonorrhea in Canada, 2010-2015. Can Commun Dis Rep 2018 Feb;44(2):37-42. DOI PubMed

5. Ling DI, Janjua NZ, Wong S, Krajden M, Hoang L, Morshed M, Achen M, Murti M, Lester RT, Wong J, Ogilvie G, Gilbert $M$. Sexually transmitted infection trends among gay or bi-sexual men from a clinic-based sentinel surveillance system in British Columbia, Canada. Sex Transm Dis 2015 Mar;42(3):153-9. DOI PubMed

6. Mohammed H, Mitchell H, Sile B, Duffell S, Nardone A, Hughes $G$. Increase in sexually transmitted infections among men who have sex with men, England, 2014. Emerg Infect Dis 2016 Jan;22(1):88-91. DOl PubMed

7. Rietmeijer CA, Patnaik JL, Judson FN, Douglas JM Jr. Increases in gonorrhea and sexual risk behaviors among men who have sex with men: a 12-year trend analysis at the Denver Metro Health Clinic. Sex Transm Dis 2003 Jul;30(7):562-7. DOl PubMed

8. Jordan AE, Perlman DC, Neurer J, Smith DJ, Des Jarlais DC, Hagan $\mathrm{H}$. Prevalence of hepa-titis $\mathrm{C}$ virus infection among $\mathrm{HIV}+$ men who have sex with men: a systematic review and meta-analysis. Int J STD AIDS 2017 Feb;28(2):145-59. DOI PubMed

9. Pakianathan M, Whittaker W, Lee MJ, Avery J, Green S, Nathan B, Hegazi A. Chemsex and new HIV diagnosis in gay, bisexual and other men who have sex with men attending sexual health clinics. HIV Med 2018 May;19(7):485-90. DOI PubMed

10. Institute of Medicine. The health of lesbian, gay, bisexual, and transgender people: build-ing a foundation for better understanding. Washington (DC): National Academies Press; 2011. https://www.ncbi.nlm.nih.gov/books/NBK64806/pdf/ Bookshelf_NBK64806.pdf

11. Weatherburn P, Hickson F, Reid DS, Marcus U, Schmidt AJ. European Men-who-have-sex-with-men Internet Survey (EMIS-2017): design and methods. Sex Res Soc Policy 2019 (in press).

12. The EMIS Network. EMIS-2017: 33 language versions of the European Men-who-have-sex-with-men Internet Survey. The EMIS Network; 2017 (Accessed 2019-09-04). http:// sigmaresearch.org.uk/tags/all/tag/EMIS-2017

13. Kroenke K, Spitzer RL, Williams JB, Löwe B. An ultra-brief screening scale for anxiety and depression: the PHQ-4. Psychosomatics 2009 Nov-Dec;50(6):613-21. DOI PubMed

14. Shields AL, Caruso JC. A reliability induction and reliability generalization study of the Cage Questionnaire. Educ Psychol Meas 2004;64(2):254-70. DOI

15. Holt M, Lea T, Mao L, Zablotska I, Lee E, de Wit JB, Prestage G. Adapting behavioural surveillance to antiretroviral-based HIV prevention: reviewing and anticipating trends in the Australian Gay Community Periodic Surveys. Sex Health 2017 Feb;14(1):72-9. DOI PubMed
16. European Centre for Disease Prevention and Control. Monitoring implementation of the Dublin Declaration. Solna (SE): ECDC. https://ecdc.europa.eu/en/all-topic s-zhiv-infection-and-aidsprevention-and-control/ monitoring-implementation-dublin

17. Ferlatte O, Dulai J, Hottes TS, Trussler T, Marchand R. Suicide related ideation and behav-ior among Canadian gay and bisexual men: a syndemic analysis. BMC Public Health 2015 Jul;15(1):597. DOl PubMed

18. Bourne A, Weatherburn P. Substance use among men who have sex with men: patterns, motivations, impacts and intervention development need. Sex Transm Infect 2017 Aug;93(5):342-6. DOl PubMed

19. Noor SW, Adam BD, Brennan DJ, Moskowitz DA, Gardner S, Hart TA. Scenes as micro-cultures: examining heterogeneity of HIV risk behavior among gay, bisexual, and other men who have sex with men in Toronto, Canada. Arch Sex Behav 2018 Jan;47(1):309-21. DOI PubMed

20. Messier-Peet M, Apelian H, Lambert G, Cox J. Chemsex \& mental health in gbMSM in Montreal: results from Engage Cycle 1. Gay Men's Health Summit, 2018. Vancouver (BC); 2018 Nov 8-9. https://www.engage-men.ca/wp-content/ uploads/2018/04/Engage-Montr\%C3\%A9al-Chemse x-Mental-Health-FINAL-ENGLISH.pdf

21. Card KG, Lachowsky NJ, Armstrong HL, Cui Z, Wang L, Sereda P, Jollimore J, Patterson TL, Corneil T, Hogg RS, Roth EA, Moore DM. The additive effects of depressive symp-toms and polysubstance use on HIV risk among gay, bisexual, and other men who have sex with men. Addict Behav 2018 Jul;82:158-65. DOl PubMed

22. Grewal R, Allen VG, Gardner S, Moravan V, Tan DH, Raboud J, Bayoumi AM, Kaul R, Mazzulli T, McGee F, Rourke SB, Burchell AN; OHTN Cohort Study Research Team. Se-rosorting and recreational drug use are risk factors for diagnosis of genital infection with chlamydia and gonorrhoea among HIV-positive men who have sex with men: results from a clinical cohort in Ontario, Canada. Sex Transm Infect 2017 Feb;93(1):71-5. DOl PubMed

23. Hart TA, Noor SW, Adam BD, Vernon JR, Brennan DJ, Gardner S, Husbands W, Myers T. Correction: Number of psychosocial strengths predicts reduced HIV sexual risk behaviors above and beyond syndemic problems among gay and bisexual men. AIDS Behav 2017 Oct;21(10):3035-46. DOI

24. Batchelder AW, Safren S, Mitchell AD, Ivardic I, O'Cleirigh C. Mental health in 2020 for men who have sex with men in the United States. Sex Health 2017 Feb;14(1):59-71. DOl PubMed

25. Armstrong $\mathrm{H}$, Pan S, Barath J, Olarewaju G, Rodrigues R, Messier-Preet M, Apelian H, Hull M, Tan D, Lachowsky N, Jollimore J, Hart T, Grace D, Lambert G, Cox J, Moore D. Trends in awareness and use of PrEP among HIV-negative men who have sex with men in Vancouver, Toronto, and Montreal. Sex Transm Infect 2019;95:A231-2. https://sti.bmj. com/content/sextrans/95/Suppl_1/A231.2.full.pdf 
26. Morgan J, Ferlatte $O$, Salway T, Wilton J, Hull M. Awareness of, interest in, and willing-ness to pay for HIV pre-exposure prophylaxis among Canadian gay, bisexual, and other men who have sex with men. Can J Public Health 2018 Dec;109(56):791-9. DOI PubMed

27. Hall EW, Heneine W, Sanchez T, Sineath RC, Sullivan P. Preexposure prophylaxis modali-ty preferences among men who have sex with men and use social media in the United States. J Med Internet Res 2016 May; 18(5):e111. DOI PubMed

28. Rapid Response Service. Facilitators and barriers to health care for lesbian, gay and bisex-ual (LGB) people. Toronto (ON): Ontario HIV Treatment Network; 2014 Mar. https://www.ohtn.on.ca/Pages/Knowledge-Exchange/ Rapid-Responses/Documents/RR79.pdf

29. Mosley T, Khaketla M, Armstrong HL, Cui Z, Sereda P, Lachowsky NJ, Hull MW, Olarewaju G, Jollimore J, Edward J, Montaner JS, Hogg RS, Roth EA, Moore DM. Trends in awareness and use of HIV PrEP among gay, bisexual, and other men who have sex with men in Vancouver, Canada 2012-2016. AIDS Behav 2018 Nov;22(11):3550-65. DOI PubMed

30. Ha S, Whyte CA, Paquette D, Tarasuk J, Latham-Carmanico C, Holt M. Comprehensive testing for sexually transmitted and blood-borne infections among men who have sex with men in Canada: findings from an enhanced surveillance system (M-Track 2005-2007). J Infect Dis 2014;25 Suppl 4:75A. https://www.cahr-acrv.ca/ wp-content/uploads/2012/10/CAHR-2014-Abstract-Book.pdf
31. Frankis J, Goodall L, Clutterbuck D, Abdul-Razak A, Flowers P. Regular STI testing amongst men who have sex with men and use social media is suboptimal - a cross-sectional study. Int J STD AIDS 2017 May;28(6):573-83. DOI PubMed

32. Holt M, Hull P, Lea T, Guy R, Bourne C, Prestage G, Zablotska I, de Wit J, Mao L. Com-prehensive testing for, and diagnosis of, sexually transmissible infections among Australi-an gay and bisexual men: findings from repeated, cross-sectional behavioural surveillance, 2003-2012. Sex Transm Infect 2014 May;90(3):208-15. DOI PubMed

33. Lourenço L, Colley G, Nosyk B, Shopin D, Montaner JS, Lima VD; STOP HIV/AIDS Study Group. High levels of heterogeneity in the HIV cascade of care across different population subgroups in British Columbia, Canada. PLoS One 2014 Dec;9(12):e115277. DOI PubMed

34. Lambert G, Cox J, Messier-Peet M, Apelian H, Moodie EE. Engage Montréal. Portrait of the sexual health of men who have sex with men in Greater Montréal: Cycle 2017-2018: Highlights. Montréal (QC): Direction régionale de santé publique, CIUSSS du Centre-Sud-de-l'île-de-Montréal; 2019 Jan. https://www.engage-men.ca/wp-content/ uploads/2019/04/Engage_Highlights_ENG_Mars-2019.pdf 